\title{
Optimizing Atomic Force Microscopy Techniques to Identify Amyloid Fibrils in the Seminal Plasma of Mammalian Species
}

\author{
Nathaly Cormier, Steve Tardif, Ozgur Yavuzcetin and Ian Ruble \\ University of Wisconsin-Whitewater, Whitewater, Wisconsin, United States
}

Amyloids are aggregates of proteins characterized by a fibrillar morphology of $\sim 7-13 \mathrm{~nm}$ in diameter, a $\beta$-sheet secondary structure and ability to be stained by particular dyes, such as thioflavin T (Fowler et al., 2012). Amyloids, which are typically associated with neurodegenerative diseases such as Alzheimer's, are also found in human seminal plasma (SP), and further studies showed that in vitro and endogenous amyloid fibrils in SP enhanced HIV transmission (Usmani et al., 2014). Additional studies in mice suggested that amyloid structures may not be just linked to diseases, but in normal physiological processes such as sperm maturation, fertilization and early development (Guyonnet et al., 2014; Sylva et al., 2015; Whelly et al., 2012; Whelly et al., 2015; Whelly et al., 2016). The main purpose of this study was to optimize the techniques of Atomic Force Microscope (AFM) and identify amyloid fibrils in SP of different mammalian species to better characterize amyloid structures in biological fluids. To identify the optimal substrate, two methods of plating were tested: 1) spin coating on silicon wafers via a spin coating apparatus and, 2) drop casting by placing $\sim 15 \mathrm{~mL}$ of bovine SP solution onto two different scanning media, silicon wafers or freshly cleaved mica. Various ratios of SP and diluents (water or PBS) were initially examined using AFM in tapping mode, but the concentration of fibrils in SP of either bull or Rhesus monkey was too low to visualize them efficiently on these surfaces. Ultimately, tangential filtration and cation exchange chromatography were used with pools of SP from several bovine ejaculates, due to the large volume required for the purification process. The latter procedure yielded a significant enrichment of endogenous amyloid structures, including fibrils. By further refining the current AFM methods to visualize and analyze amyloids using Nanoscope Analysis Software, the characterization of amyloid structures and other protein aggregates within biological fluids will surely be improved. Eventually, results from this study may help to develop new strategies for treating HIV and similar sexually transmitted diseases by inhibiting the formation of amyloid fibrils.

References

Fowler et al., 2012; Usmani et al., 2014; Guyonnet et al., 2014; Sylva et al., 2015; Whelly et al., 2012; Whelly et al., 2015; Whelly et al., 2016. 